\title{
IntervenÇấo Mediada Por Pares no Engajamento Acadêmico de Alunos COM AUTISMO ${ }^{1,2}$ \\ PeER Mediated InterVEntion in the Academic ENGagement of Students WITH AUTISM
}

\author{
Fabiane dos Santos RAMOS 3 \\ Daniele Denardin de BITTENCOURT ${ }^{4}$ \\ Síglia Pimentel Höher CAMARGO5 \\ Carlo SCHMIDT ${ }^{6}$
}

\begin{abstract}
RESUMO: A literatura vem destacando a fragilidade das práticas pedagógicas de professores de alunos com autismo. Desse modo, este estudo propôs-se a investigar o efeito da Intervençáo Mediada por Pares (IMP) sobre o engajamento acadêmico de alunos com autismo. A IMP tem se mostrado promissora por relatar resultados animadores no desenvolvimento de habilidades desses educandos no contexto escolar. Apesar dessa intervenção mostrar fartas evidências na promoção de habilidades sociais, poucos estudos investigam sua efetividade quanto ao engajamento em atividades acadêmicas. Participaram desta investigação dois alunos com autismo dos anos iniciais do Ensino Fundamental, quatro colegas de sala de aula e uma educadora especial. Os comportamentos mediadores dos pares e o engajamento do aluno com autismo em tarefas acadêmicas foram registrados e analisados por meio de filmagens e de um delineamento AxB. Os resultados mostraram que a IMP aumentou o comportamento mediador dos pares e o tempo de engajamento dos alunos com autismo em tarefas acadêmicas de sala de aula. Implicações práticas e relevância desses achados também são discutidos.
\end{abstract}

PALAVRAS-CHAVE: Autismo. Intervenção Mediada por Pares. Escolarização.

ABSTRACT: Literature has highlighted the fragility of the pedagogical practices of teachers of students with autism. Thus, this study aimed to investigate the effect of the Peer-Mediated Intervention (PMI) on the academic engagement of students with autism. The PMI has been promising for reporting encouraging results in the skill development of these students in the school context. Although this intervention shows strong evidence in the promotion of social skills, few studies investigate its effectiveness in engaging in academic activities. Two students with autism from the first grades of Elementary School, four classmates and an educator of Special Education participated in the study. Peer mediating behaviors and student with autism engagement in academic tasks were recorded and analyzed through filming and AxB design. The results showed that the PMI increased the engagement time of students with autism in academic tasks of the classroom. Practical implications and relevance of these findings are also discussed.

KEYWORDS: Autism. Peer-Mediated Intervention. Schooling.

\footnotetext{
${ }^{1}$ https://doi.org/10.1590/1980-54702021v27e0261

${ }^{2}$ Este artigo decorre de pesquisa financiada pelo Conselho Nacional de Desenvolvimento Científico e Tecnológico - CNPq (Processo 306379/019-8 - Bolsista Produtividade).

${ }^{3}$ Doutora em Educação pela Universidade Federal de Santa Maria (UFSM). Especialista em Educação Inclusiva pela Fundaçăo Getúlio Vargas (FGV). Educadora Especial pela UFSM. Integrante do Grupo de Pesquisa Educação Especial e Autismo (EdEA/UFSM). Santa Maria / Rio Grande do Sul / Brasil. E-mail: fabianeramos.3110@gmail.com. ORCID: https://orcid.org/0000-0001-9778-5913

${ }^{4}$ Mestre em Educação (UFSM). Educadora Especial (UFSM). Santa Maria / Rio Grande do Sul / Brasil. E-mail: danieledenardinbittencourt@yahoo.com.br. ORCID: https://orcid.org/0000-0002-5150-2663

${ }^{5}$ Professora Associada do Programa de Pós-Graduação em Educação da Universidade Federal de Pelotas (UFPel). Coordenadora do Grupo de Estudos e Pesquisas e Autismo e Inclusão (GEPAI/UFPel). Pelotas / Rio Grande do Sul / Brasil. E-mail: sigliahoher@ yahoo.com.br. ORCID: https://orcid.org/0000-0001-7058-6519

${ }^{6}$ Professor Associado do Programa de Pós-Graduação em Educação da UFSM. Coordenador do Grupo de Pesquisa Educação Especial e Autismo (EdEA/UFSM). Santa Maria / Rio Grande do Sul / Brasil. E-mail: carlo.schmidt@ufsm.br. ORCID: https:// orcid.org/0000-0003-1352-9141
} 


\section{INTRODUÇÁo}

A legislação brasileira orienta que a escolarização de alunos com autismo seja realizada em escolas regulares em todas as modalidades e em todos os níveis de ensino, juntamente a seus colegas de classe, de acordo com a Lei Brasileira de Inclusão da Pessoa com Deficiência - Lei no 13.146, de 6 julho de 2005. Assim sendo, pesquisadores direcionam seus estudos na busca de alternativas pedagógicas e de intervençóes que possam auxiliar e suprir as necessidades educacionais desses alunos (Ganz \& Flores, 2008; Harper et al., 2008), levando em consideração a dinâmica do espaço escolar.

Alunos com o diagnóstico de Transtorno do Espectro Autista (TEA) apresentam comprometimentos em níveis variados, tendo como características principais dificuldades na interação social e na comunicação (Associação Americana de Psicologia [APA], 2013). Tais características podem vir associadas a alteraçóes sensoriais e comportamentais do aluno, o que tende a agravar os seus sintomas, necessitando de apoio maior em seu desenvolvimento.

As dificuldades oriundas do diagnóstico do TEA tomam uma proporção mais significativa na escola, quando, com o passar do tempo, as habilidades acadêmicas do aluno com TEA não se desenvolvem. Tal evidência mostra-se como um desafio aos docentes que precisam, entre outras funçóes, promover de forma qualitativa o desenvolvimento das habilidades de aprendizagem desses alunos.

Frente a isso, a literatura tem apontado a fragilidade com que se desenvolve o processo de escolarização dos alunos com TEA (Camargo \& Bosa, 2009; Martins, 2007), bem como tem mostrado indícios dos fatores que contribuem para tal fragilidade. Dentre eles, destacam-se o pouco conhecimento docente acerca das idiossincrasias do transtorno; as dificuldades dos professores em adequar metodologias coerentes com as necessidades dos alunos (Lemos et al., 2014; Nunes et al., 2013); a dinâmica do espaço escolar frente às dificuldades de interação e comunicação desses alunos; entre outros.

A Intervenção Mediada por Pares (IMP) mostra-se como uma alternativa frente a esse cenário. Trata-se de uma intervenção que utiliza os pares típicos - colegas de classe - para promover a mediação junto à criança com autismo, auxiliando de forma qualitativa o desenvolvimento de habilidades importantes anteriormente elencadas pela equipe que atende a esses alunos (Ramos et al., 2018). Os pares exercem um papel de agentes de intervenção, auxiliando os alunos alvo na realização de trocas sociais e, assim, influenciam tanto em seu desenvolvimento quanto em seu desempenho escolar (Carter et al., 2010; Gifford-Smith \& Brownell, 2003; Rubin et al., 2009). Sua implementação envolve etapas prévias ao início da intervenção, como a escolha e o treinamento dos pares, geralmente sendo realizado pelo professor de sala regular concomitante com o trabalho do educador especial.

Por apresentar evidências significativas de ganhos de habilidades nos alunos com TEA, esta tem sido uma estratégia muito usada em um cenário internacional (Goldstein et al., 1992; Kamps, et al., 1992; McConnell, 2002; Odon et al., 1993; Ostrosky \& Kaiser, 1995; Pierce \& Schreibman, 1997; Rogers, 2000; Strain et al., 1995; Watkins et al., 2015). Diversos estudos estrangeiros já demonstram que essa intervenção auxilia no desenvolvimento e na promoção de habilidades sociais, ampliando a conversação, o contato visual e as trocas recíprocas (Ganz \& Flores, 2008; Ganz, et al., 2012; Jansen et al., 2003), bem como têm mostrado in- 
dicativos de resultados satisfatórios relacionados a habilidades acadêmicas (Chan et al., 2009; Kamps et al., 2002; Watkins et al., 2015).

Assim, por tratar-se de uma intervenção de fácil aplicação e coerente com ambientes naturalísticos, a IMP tem se destacado como uma estratégia viável para ser implementada nas escolas brasileiras (Schmidt et al., 2019). Os ganhos relacionados a ela tendem a ser mantidos, tornando-se permanentes, justamente por ser desenvolvida em um ambiente em que a criança convive diariamente.

Apesar de ampla divulgação e comprovação de resultados no âmbito internacional, nacionalmente pouco se tem falado sobre essa intervenção. Dentre os estudos encontrados, Ramos et al. (2018) realizaram uma revisão teórica sobre a IMP na educação de alunos com autismo e suas implicaçóes para a pesquisa e para a prática pedagógica, contribuindo para o entendimento da origem da intervenção e auxiliando professores em sua implementação nas escolas, mas sem dados referentes à efetividade da sua aplicação.

Somente dois estudos de intervenção nacionais foram encontrados, ambos para o ensino de habilidades sociais. Bastos et al. (2018) efetivaram uma pesquisa prática envolvendo a modelação em vídeo no ensino de pares típicos como estratégia para promover habilidades sociais em crianças com autismo. Com a participação de duas crianças de 6 anos de idade, diagnosticadas com TEA, e seis crianças, da mesma idade, com desenvolvimento típico, em sessóes de brincadeira livre durante 15 minutos, puderam comprovar o aumento no número de respostas de "iniciar interação" e "responder interação", a partir da mediação dos pares.

Já Carvalho et al. (2017) investigaram a IMP como possibilidade de ensinar habilidades sociais para quatro crianças com autismo, em escolas regulares. Inicialmente, o contato das crianças com os 12 colegas de classe foi sem instrução específica, com o objetivo de verificar como ocorria a interação entre eles. Em seguida, os pares típicos desenvolveram estratégias para relacionarem-se com seus colegas. Os resultados apontaram efetivo aumento de interação entre eles, a partir do início da intervenção.

Apesar de as pesquisas nacionais apontarem resultados práticos e promissores, ainda há escassez de estudos relacionados à efetividade da IMP no desenvolvimento da aprendizagem acadêmica dos alunos com autismo em escolas públicas brasileiras. Assim sendo, esta pesquisa teve o objetivo de investigar o efeito da Intervenção Mediada por Pares sobre o engajamento acadêmico de alunos com autismo. O intuito é contribuir para a ampliação de dados que aportem o uso dessa intervenção em um cenário nacional.

\section{Método}

Foi utilizado um delineamento de caso único intra-sujeitos ${ }^{7}$ do tipo AxB para avaliar o efeito da IMP no comportamento mediador dos pares e no engajamento na tarefa (Nunes \& Walter, 2014). Participaram dois alunos com autismo, João e Maria (nomes fictícios) que atenderam aos seguintes critérios de inclusão: a) ter diagnóstico clínico de TEA; b) estar matri-

\footnotetext{
${ }_{7}^{7}$ Outras denominações também são encontradas na literatura nacional e internacional: delineamento de sujeito com seu próprio controle; delineamento de pesquisa $\mathrm{N}=1$; delineamento de sujeito único ou caso único; delineamento intensivo; single case design; e single subject design (Sampaio et al., 2008).
} 
culado nos anos iniciais do Ensino Fundamental, c) não participar de outras intervenções nem utilizar medicamentos.

João, com 7 anos e 5 meses, frequentava o $2^{\circ}$ ano do Ensino Fundamental e recebeu o diagnóstico de autismo aos 5 anos. $\mathrm{O}$ aluno apresentava dificuldades no momento da alimentação e para realizar hábitos de higiene com independência. A linguagem era compreensível, porém não clara. Não apresentava comportamentos agressivos ou autolesivos. Obteve escore de 34,5 pontos por meio da Childhood Autism Rating Scale (CARS), o que equivale à autismo leve a moderado. Em relação às aprendizagens, reconhecia as letras de seu nome e algumas vogais, o que, de acordo com Ferreiro \& Teberosky (1999), caracteriza a fase pré-silábica de alfabetização.

Já Maria é uma menina com 6 anos e 10 meses de idade e frequentava o $1^{\circ}$ ano do Ensino Fundamental. Recebeu o diagnóstico aos 5 anos, sendo constatado autismo associado à Síndrome de West ${ }^{8}$. Ela é dependente de auxílio para vestir-se e locomover-se e não desenvolveu a linguagem verbal. Obteve escore de 44 pontos na escala CARS, o que caracteriza um quadro de autismo grave. Nas aprendizagens, não diferenciava letras de símbolos, configurando a fase pré-silábica de alfabetização (Ferreiro \& Teberosky, 1999).

Além disso, quatro crianças com desenvolvimento típico foram selecionadas para atuarem como pares na intervenção, de acordo com a Ficha de Seleção dos Pares (Ramos, 2019), respondida por duas professoras - de João, foi a professora regente que o acompanhou no ano anterior - primeiro ano do Ensino Fundamental; e de Maria, a professora da Educação Infantil. Uma educadora especial participante possuía Licenciatura Plena em Educação Especial, atuava na escola há três anos em turnos de 20 horas semanais e atendia os alunos com autismo em Atendimento Educacional Especializado (AEE), atuando também como orientadora dos pares típicos.

\subsection{INSTRUMENTOS ${ }^{9}$}

Os instrumentos de coleta de dados utilizados nesta pesquisa foram:

- Childhood Autism Rating Scale - CARS (Pereira, 2007): validado para a realidade brasileira por Pereira et al. (2008). Foi utilizado na pontuação da intensidade dos sintomas do autismo; aplicado pela assistente de pesquisa e pela educadora especial, que receberam treinamento prévio. Teve resultado considerado a partir da média das pontuaçóes realizadas entre ambas.

- $\quad$ Ficha de caracterização dos alunos com autismo (Ramos, 2019): foram coletados os dados sociodemográficos e o perfil comportamental e educacional dos alunos com autismo, sendo composto por seis seções: 1) Dados gerais sobre a escolarização; 2) Características clínicas; 3) Comportamentos; 4) Habilidades sociais; 5) Habilidades pré-acadêmicas e acadêmicas; e 6) Habilidades de linguagem e comunicação. Foi respondida pela educadora especial da escola e aplicada pela assistente de pesquisa (segunda autora deste artigo).

\footnotetext{
${ }^{8}$ Síndrome de West: síndrome neurológica, sendo um tipo raro de epilepsia que causa convulsōes, apresentando uma tríade de sintomas: os espasmos infantis, o retardo no desenvolvimento neuropsicomotor e a hypsarritmia (súbitas eclosóes de atividades elétricas, algumas com alteraçóes de alto potencial no eletroencefalograma). Os abalos são acompanhados de falta de consciência e espasmos, causando alteraçóes motoras, sendo a mais frequente a hipotonia (Lux \& Osborne, 2004).

${ }^{9}$ Com exceção da escala (CARS), os instrumentos utilizados neste estudo foram elaborados pelo Grupo de Pesquisa Educação Especial e Autismo da UFSM.
} 
- $\quad$ Ficha de seleção de pares (Ramos, 2019): integra o protocolo para a implementação da IMP (o Peer Selection Checklist, de Sam \& Afirm Team, 2015). Foi traduzida e retrotraduzida (inglês-português-inglês) e comparada ao original para ajustes. A ficha sugere entre dois e seis colegas para cada aluno com autismo e traz 11 critérios de escolha a serem pontuados em uma Escala Likert de zero a cinco pontos, tais como faixa etária próxima ao colega, bom rendimento escolar, domínio de habilidades sociais e de linguagem.

- $\quad$ Protocolo de observação dos comportamentos mediadores dos pares (Ramos, 2019): mede a ocorrência de comportamentos dos pares na tarefa de mediar as aprendizagens da criança com autismo por meio de redirecionamento e encorajamento do colega com autismo para a tarefa. $\mathrm{O}$ redirecionamento divide-se em: mandos verbais, prompts (comandos) verbais, físicos ou gestuais e modelagem. Já o encorajamento divide-se em: incentivadores e feedback.

- $\quad$ Protocolo de pontuação de engajamento na tarefa (Ramos, 2019): subdividido em aprendizagem pré-acadêmica (engajamento na tarefa e fora da tarefa). O protocolo permitia marcar "engajado na tarefa" (ET) ou "fora da tarefa" (FT) a cada intervalo de tempo de dez segundos. Cada variável foi coletada em um sistema intervalar diferente, a partir da observação de vídeos por dois avaliadores independentes. Foi considerado ET se a criança permanecesse durante dez segundos na tarefa e FT quando comportamentos ocorressem a partir de cinco segundos, durante o intervalo. Portanto, conceitualmente, o aluno foi considerado engajado quando mantinha o foco de atenção na tarefa, no seu colega ou nos materiais colocados sobre a mesa, permanecia sentado em sua classe e manipulava os materiais pedagógicos (por exemplo, lápis e folha) conforme proposto na tarefa, durante dez segundos.

\subsection{Procedimentos E ANÁlise dos dados}

Os vídeos utilizados na intervenção foram coletados na escola onde os alunos estudavam, produzidos a partir de filmagens realizadas duas vezes por semana durante 20 minutos, ao longo de seis meses. Cada sessão de 20 minutos foi subdividida em quatro recortes de cinco minutos, sendo sorteados randomicamente um desses segmentos para codificação do protocolo por dois avaliadores.

Para a filmagem, foi utilizada uma câmera móvel de vídeo instalada na sala de aula, enfocando o local onde os alunos com autismo estavam. As filmagens aconteceram em dias e horários diferentes, combinados previamente com a professora para ocorrerem durante as atividades pedagógicas, que esta propunha em seu plano de aula, realizadas nas mesas da sala de aula dos alunos. Foram produzidas 18 sessóes de vídeos de intervenção para João e 14 para Maria, seguindo as recomendaçóes éticas para pesquisas envolvendo seres humanos, com o projeto aprovado pelo Comitê de Ética em Pesquisa CAAE: 66643117.9.0000.5346 com o Parecer no. 2.024.648.

$\mathrm{Na}$ etapa de linha de base, os pares estavam presentes lado a lado com os participantes, mas sem intervenção ou orientação específica. Cada participante teve sua linha de base encerrada quando houve estabilidade dos dados entre as sessóes com uma variância menor que 50\%, sem altos picos de variabilidade (Byers et al., 2012). 
A etapa de intervenção iniciou com a orientação aos pares típicos, que obedeceu a seguinte sequência: 1) Foram observados e discutidos os vídeos entre a equipe de pesquisa e as educadoras especiais da escola, no intuito de avaliar as estratégias utilizadas pelos pares; 2) Foram feitos encontros na sala de recursos multifuncionais da escola, pouco antes de cada intervenção, entre a educadora especial e os pares, para orientação sobre estratégias nas sessóes anteriores e planejamento das seguintes. Imediatamente, antes da próxima sessão de intervenção, os pares eram relembrados sobre os objetivos para aquele momento.

$\mathrm{Na}$ intervenção na sala de aula, os colegas posicionaram-se entre os alunos com autismo, cada um de um lado. Essa organização, além de minimizar as esquivas à tarefa (levantar-se e sair da classe), potencializou as atividades de interação entre os trios. Assim, os pares mediaram a realização das atividades pedagógicas propostas pelas professoras junto aos alunos com autismo (chamando sua atenção para retomar/manter o contato visual na tarefa e retirar da mesa os materiais não essenciais para a realização da tarefa, mostrando/apontando para o objetivo da tarefa, reforçando respostas corretas).

Os dados sobre o comportamento mediador dos pares e o engajamento na tarefa foram graficamente representados, com o objetivo de analisar, de forma individualizada e visual, o impacto da intervenção nas variáveis investigadas. Além da inspeção visual e comparativa do gráfico quanto à variabilidade média e à tendência dos dados intra e inter fases do estudo (linha de base e intervenção), esses dados também foram estatisticamente analisados. Foi calculado o Tau-U por meio de um software online $e^{10}$, que se refere à medida da magnitude de mudança entre as fases de linha de base e a intervenção para delineamentos de caso único, para avaliar sua significância estatística $(\mathrm{p} \leq 0,05)$ e os respectivos intervalos de confiança (CI 90\%) (Parker \& Vannest, 2012). O Tau-U ${ }^{11}$ é um índice que sumariza a eficácia da intervenção e avalia o quanto os dados não coincidem com os da linha de base. O omnibus effect size foi calculado para a obtenção de uma medida geral do estudo, considerando o conjunto das mudanças ocorridas para cada participante.

Os vídeos das sessóes foram analisados individualmente. Em casos de divergência na pontuação acima de 40\%, os avaliadores reavaliavam conjuntamente os segmentos analisados, buscando um consenso. Quanto aos comportamentos mediadores dos pares, os indices de concordância entre os juizes resultaram um escore total de confiabilidade entre os observadores de 99,7\% para o protocolo de Maria e 98,7\% para o protocolo de João. Já para o comportamento de engajamento na tarefa, foi obtido $99 \%$ na pontuação do protocolo da Maria e $72 \%$ na pontuaçáo do protocolo de Joáo.

Avaliou-se a fidelidade da intervenção para medir o quanto a IMP foi conduzida conforme o planejado, utilizando-se o Protocolo de fidelidade da intervenção (Sam \& Afirm Team, 2015). Os escores indicaram uma fidelidade de $84 \%$ na intervenção com Maria e $80 \%$ com João.

A validade social do estudo foi realizada por intermédio da aplicação de três questionários destinados aos professores, à gestão escolar e às mães dos participantes. A análise da

\footnotetext{
${ }^{10}$ Ver mais informaçôes em: www.singlecaseresearch.org. Acesso em: 18 maio 2021.

${ }^{11}$ Seus escores variam de 0 a 1 (por exemplo, um escore de 0,57 indica que 57\% dos dados não estáo sobrepostos, o que revela o percentual de melhora entre as fases). A tendência é considerar que escores de Tau-U representam entre 0 e 0.2 - pequeno efeito; entre 0.2 e 0.8 - moderado a grande efeito; entre 0.8 e 1 - muito grande o efeito (Parker et al., 2011).
} 
validade indicou que a IMP, na percepção dos envolvidos, foi efetiva na aprendizagem dos alunos com autismo em contexto de inclusão escolar, favorecendo seu desenvolvimento e, consequentemente, sua aprendizagem em diferentes contextos.

\section{Resultados}

A Figura 1, a seguir, apresenta gráficos sobrepostos, os quais evidenciam os efeitos do comportamento mediador dos pares (redirecionamento e encorajamento) sobre o comportamento de engajamento na tarefa (ET/FT) dos alunos Joáo e Maria. Os resultados resumem de forma geral o processo interventivo realizado, considerando as duas variáveis investigadas no estudo. Por inspeção visual, é possível perceber um aumento tanto da frequência de comportamento mediador dos pares quanto do percentual do engajamento na tarefa (ET) após o início da intervenção para ambos os participantes, bem como é notável a variabilidade no desempenho das intervençóes, com picos de altos e baixos, explicada por particularidades em cada sessão. 


\section{Figura 1}

Comportamento mediador dos pares sobre o engajamento na tarefa

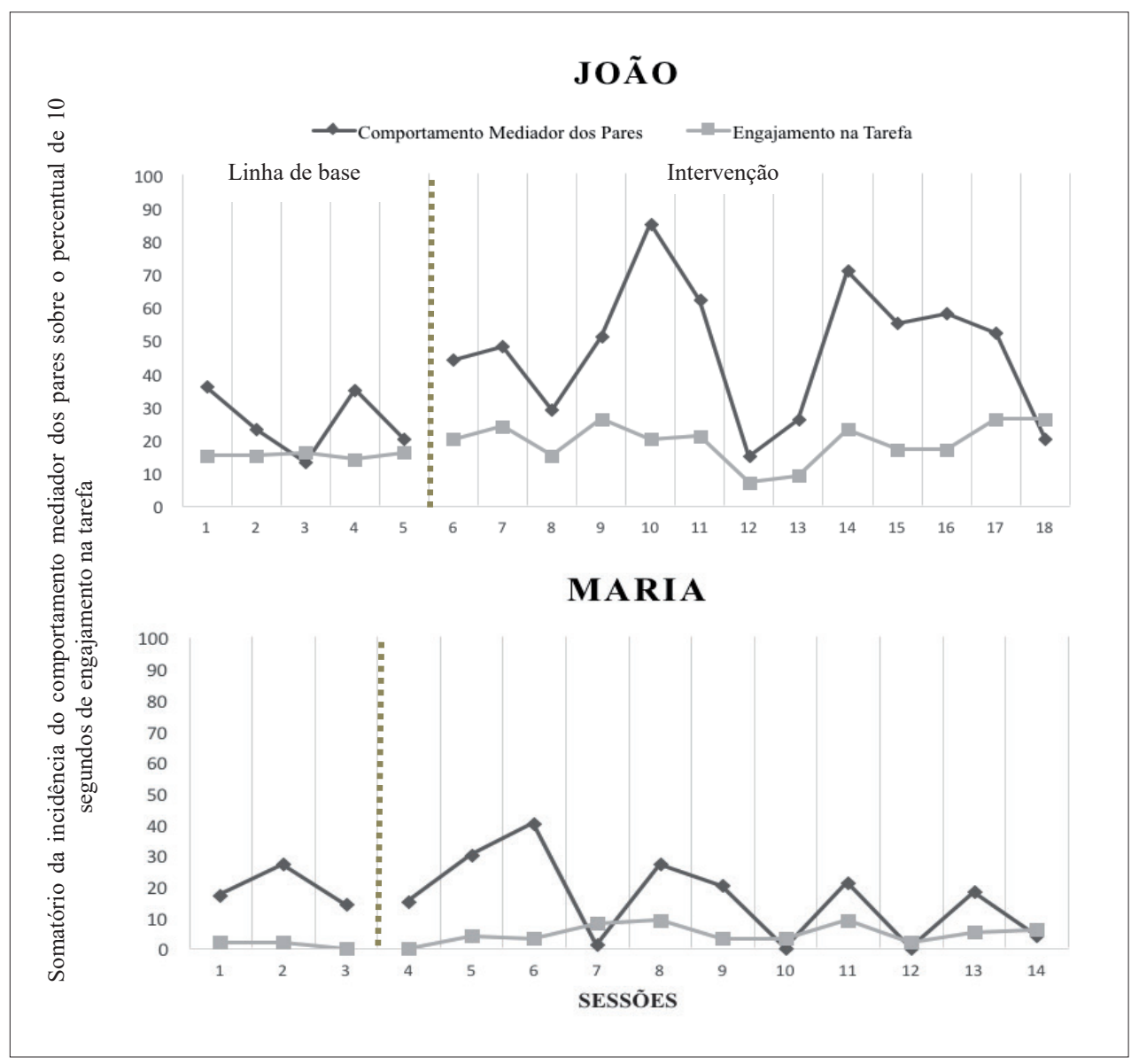

A média do comportamento mediador dos pares na intervenção para João $(m=33,34$; $d p=20,57)$ e Maria $(m=10,36 ; d p=11,14)$ foi superior à observada na linha de base $(m=20,30$; $d p=8,53$ e $m=10,33$; $d p=10,78)$, respectivamente com um maior incremento observado para o participante João. Em relação ao percentual de comportamentos de engajamento na tarefa também se verifica um aumento na frequência entre as medidas da linha de base $(m=1,33$; $d p=1,15$ e $m=15,20 ; d p=0,83)$ e da fase de intervenção $(m=4,7 ; d p=2,95$ e $m=19,15 ; d p=6,20)$ para João e Maria, respectivamente. Esses dados ilustram que os participantes passaram a dispender uma quantidade maior de tempo engajados nas atividades acadêmicas em sala de aula a partir da intervenção. A Tabela 1 que segue indica a magnitude dessas mudanças a partir do cálculo do Tau-U. 


\section{Tabela 1}

Tau-U para comportamento mediador dos pares e engajamento na tarefa

\begin{tabular}{l|l|l|l|l|l|l}
\hline \multicolumn{1}{c|}{ Variável } & \multicolumn{3}{c|}{ Comportamento mediador dos pares } & \multicolumn{3}{c}{ Engajamento na tarefa } \\
\hline Participante & Tau-U & P Value & CI $90 \%$ & Tau-U & P Value & CI $90 \%$ \\
\hline João & 0,6462 & 0,0384 & $0,133<>1$ & 0,600 & 0,0546 & $0,087<>1$ \\
\hline Maria & 0,0303 & 0,9379 & $-0,671<>0,610$ & 0,7878 & 0,0430 & $0,148<>1$ \\
\hline Omnibus effect size & 0,3814 & 0,1173 & $-0,019<>0,782$ & 0,6735 & 0,0057 & $0,273<>1$ \\
\hline
\end{tabular}

O comportamento mediador dos pares para João obteve uma melhora de $64 \%$ em relação à linha de base, com diferença estatisticamente significativa $(\mathrm{p} \leq 0.05)$. É possível inferir que esse aumento na mediação dos pares possa ter contribuído para o maior engajamento de João nas tarefas escolares na fase da intervenção, a qual difere em $60 \%$ em relação à linha de base, com diferença estatística igualmente significativa $(\mathrm{p} \leq 0.05)$.

Para Maria, observa-se um discreto aumento de 3\% no comportamento mediador dos pares em relaçáo à linha de base, dado que estatisticamente (a ser discutido quanto à relevância social) não apresenta diferença significativa. Embora o aumento na mediação dos pares para Maria tenha sido pouco expressivo, observa-se um engajamento de $78 \%$ maior na tarefa na fase de intervenção em relação à linha de base, com diferença estatisticamente significativa $(\mathrm{p} \leq 0.05)$.

Considerando os dados gerais do estudo, o resultado do omnibus effect size indica uma melhora de $38 \%$ no comportamento mediador dos pares na intervenção, sendo este escore considerado de efeito moderado, e, embora reitere os ganhos verificados ao longo da intervenção, não se mostra estatisticamente significativo $(\mathrm{p} \geq 0,05)$. Já em relação ao engajamento na tarefa, o efeito geral do estudo indica uma melhora de $67 \%$, sendo este escore considerado de grande efeito com uma relevante significância estatística da diferença encontrada $(\mathrm{p} \leq 0,05)$.

\section{Discussão}

A IMP produziu efeitos sobre o comportamento mediador dos pares e o engajamento na tarefa dos dois participantes. Os efeitos, porém, não se mostraram constantes em todas as sessōes, havendo variação ao longo da intervençáo. Os resultados indicaram aumento do engajamento ainda na primeira sessão em ambos os participantes.

No caso de Maria, embora a ascendência da linha nos gráficos seja em menor proporçáo, o aumento de comportamentos essenciais ao contexto escolar, como manter-se sentada e manusear materiais pedagógicos, por exemplo, aumentaram consideravelmente. Os episódios de maior engajamento na tarefa de Maria aconteceram na fase de intervençáo entre as sessóes 6 e 8, seguindo tendência igualmente observada entre as sessóes 10 e 11. Essa ascensão pode ser explicada em funçáo de que nessas sessóes Maria se encontrava mais calma, permitindo que os pares mediassem as atividades realizadas da forma como foram orientados, ou melhor, se utilizando de comportamento mediador de acordo com as características da aluna, auxiliando no engajamento na tarefa. Além disso, a natureza das atividades parece ter contribuído para 
seu maior engajamento, uma vez que envolviam pintura de imagens e construção de um Papai Noel para colorir e montar, trazidas pela professora.

As quedas na linha de engajamento na tarefa, verificadas nas sessóes 9 e 12, estavam relacionadas ao comportamento de não permanecer sentada, o que fez com que os pontuadores marcassem mais fora da tarefa nessas sessóes. Embora a mediação dos pares ampliasse seu tempo de permanência na classe, o que evidencia possíveis efeitos da intervenção, ela ainda manteve esse comportamento em algumas sessóes, em menor frequência.

No caso de João, os gráficos indicaram um aumento do engajamento ao longo da intervenção, com comportamentos que ampliaram a possibilidade de consolidação de aprendizagem, como o aumento de tempo dedicado à tarefa e a maior concentração e atenção. Observaram-se aumentos significativos entre as sessóes 5 e 7 , seguido de uma queda na sessão 8 , com a retomada de ascendência em seguida. Novamente, a natureza da atividade parece ser uma explicação plausível, uma vez que, na sessão 8, esta era muito abstrata e exigia do aluno habilidades de coordenação motora fina que ele não as tinha consolidadas. A frustração por não conseguir realizar a atividade fez com que o aluno ficasse agitado, permanecendo mais tempo em pé, o que resultou na baixa frequência de engajamento nessa sessão.

A queda abrupta observada na sessão 12 indica a consecução de uma tarefa razoavelmente complexa em que os alunos deveriam montar um painel a partir dos materiais de reciclagem disponibilizados pela professora. Essa demanda fez com os pares tivessem que se concentrar na realização de suas próprias atividades, além de mediar a realização da tarefa de João. Assim, o aluno recebeu menor mediação para o engajamento na tarefa em razáo de que os pares dispuseram a atenção na sua própria tarefa.

A ascendência da curva nas sessóes 9 e 14 envolveram atividades com uso de material concreto e apoios visuais, como escrever o nome em uma folha e cobrir as letras com o uso de massa de modelar. No caso de João, isso contribuiu tanto para realização da tarefa quanto se mostrou como recurso para facilitar a mediação dos pares.

A literatura sobre as melhores práticas para o autismo é vasta em apontar as atividades de natureza visual e concreta como adequadas e favoráveis para a compreensão e aprendizagem de alunos com autismo (Fialho, 2018; Ganz \& Flores, 2008; Meadan et al., 2011). Desse modo, comportamentos da criança com autismo, decorrentes da dificuldade com atividades complexas e pouco compreensíveis, tendem a gerar dificuldades na mediação dos pares, influenciando sua efetividade para o engajamento na tarefa, conforme indicam os resultados deste estudo. Verificou-se uma relação entre atividades mais complexas e abstratas com a menor mediação dos pares paralelamente ao decréscimo no tempo de engajamento na tarefa.

No que tange à influência das características dos pares no comportamento mediador, é importante considerar, por um lado, sobretudo em relação aos pares de Maria, que se tratava de crianças menores e com dificuldades de lidar com problemas de comportamento de crianças com autismo. Por outro lado, destaca-se a habilidade das crianças em utilizar a linguagem corporal nas interações, contribuindo significativamente para o andamento da intervenção, dado que o aluno Joáo, ao ser tocado pelo colega (a mão do colega no ombro ou segurando sua mão), fazia com que pouco se distraísse, mantendo a atenção por mais tempo na tarefa e, 
consequentemente, aumentando seu tempo de engajamento. Além disso, os pares de João, por terem comportamentos mais espontâneos, comemoravam as conquistas do colega mais seguidamente, dando retorno a ele sobre o que estava sendo feito. Dessa forma, o aluno com autismo, ao ser motivado por seus acertos, conseguia finalizar a tarefa dada, muitas vezes de forma correta. Charlop et al. (2018) enfatizam a importância do reforço positivo na intervenção, já que os elogios e o feedback sobre o que está sendo feito contribuem para que o comportamento desejado da criança alvo, no caso os alunos com autismo, permaneça e se amplie.

Outra questão importante de ser considerada é que os participantes do estudo possuíam faixas etárias e níveis de comprometimento diferentes, o que naturalmente se reflete em diferentes ganhos decorrentes da intervenção. Todavia, conforme já sinalizado pela literatura sobre IMP, percebe-se que a intervenção, mesmo sendo utilizada com crianças com o diagnóstico de autismo em diferentes espectros, pode trazer benefícios por meio da interaçáo com os pares, aumentando em algum nível as habilidades vitais de desenvolvimento, comumente em defasagem como a interação social e o contato visual (Hoff \& Robinson, 2002; McCurd $\&$ Cole, 2014), bem como outras habilidades específicas a serem investigadas, por exemplo, a comunicação por intermédio de sintetizadores de voz para crianças minimamente verbais (O’Donoghue et al., 2021). A partir deste estudo, percebe-se que crianças severamente acometidas, como no caso de Maria, e se salvaguardadas as condiçóes contextuais favoráveis, também podem beneficiar-se da intervenção com ampliação de possibilidades de interação social, aprendizagem por trocas, modelagem com outras crianças, resultando em maior engajamento nos comportamentos pré-acadêmicos, como permanecer sentada, saber manusear material pedagógico, entre outros. Já crianças com menor comprometimento e com a presença de linguagem oral bem desenvolvida, como no caso de João, podem beneficiar-se da interação, incorporando repertórios de linguagem, e, consequentemente, sentindo-se entusiasmadas no desenvolvimento de suas tarefas, refletindo na qualidade de sua aprendizagem acadêmica e em maior tempo de atenção e concentração nas atividades escolares.

$\mathrm{O}$ aumento da interação e consequente ampliação do engajamento na tarefa observados neste estudo, amplia e confirma os resultados da intervenção relacionada a habilidades sociais, já verificadas em outros estudos (Banda et al., 2010; Keen et al., 2011) e corrobora a assertiva de que a IMP, mesmo sendo utilizada para fins específicos, pode promover habilidades relacionadas à teoria da aprendizagem social, na qual está ancorada. Assim, as trocas estabelecidas entre crianças da mesma idade, desde que mediadas sob orientação específica, refletem em seu desempenho escolar, proporcionando ganhos mais rápidos em comportamentos anteriormente pouco desenvolvidos. O’Donoghue et al. (2021) já indicam que a simples presença dos pares é insuficiente para obter resultados promissores nas habilidades desejadas. Isso demonstra a importância da IMP como protocolo de orientação aos pares com o uso de recursos já conhecidamente efetivos para crianças com autismo, como o reforço e feedback positivo, redirecionamento, modelagem etc. (Ramos et al., 2018).

Por fim, os resultados deste estudo indicam a importância de discutir-se a IMP para a aprendizagem acadêmica, considerando-se o contexto em que a intervenção ocorre, para além da natureza das atividades propostas e as características individuais de alunos com autismo e seus pares. Foi verificado, para ambos os participantes, a importância de uma estruturação no ambiente que favoreça a mediação dos pares e o engajamento na tarefa dos participantes com 
autismo. Especialmente para Maria, que possuía um comprometimento maior e seus pares eram menores, os arranjos ambientais podem ser decisivos. A estruturação dos materiais de trabalho na classe, mantendo sob a mesa apenas aqueles para utilidade imediata na tarefa, por exemplo, minimizou distraçóes, aumentou não só o foco na atividade e o tempo na tarefa, como também favoreceu a aprendizagem funcional dos objetos, como o lápis.

Já o engajamento na tarefa de João, considerando os objetivos escolares e o seu nível de desenvolvimento, ampliou sua aprendizagem formal. A estruturação e a organização das atividades de João possibilitaram maior compreensão por parte do aluno e maior estímulo para a realização das atividades, resultando maior engajamento seu. A importância da estruturação do ambiente para o engajamento dos alunos com autismo é consistente com a literatura na área do TEA que enfatiza a necessidade de se criarem estratégias de organização e sistematização do ambiente escolar (Giaconi \& Rodrigues, 2014). Há de considerar-se, aqui, as características de adesão a rotinas e padróes de comportamentos ritualizados e inflexíveis, comumente observados em alunos com autismo.

\section{CONSIDERAÇÓES FINAIS}

No contexto educacional brasileiro, em que o processo de inclusão de crianças com autismo tende a gerar insegurança nos docentes quanto aos seus processos de ensino e de aprendizagem (Camargo et al., 2020), delimitou-se como objetivo geral deste estudo investigar os efeitos da intervenção mediada por pares nas aprendizagens pré-acadêmica e formal de alunos com autismo. Por meio do cálculo do TAu-U, foi possível observar o efeito estatístico da IMP, tanto dos comportamentos mediadores dos pares quanto do engajamento na tarefa de forma expressiva, especialmente com a aluna Maria. Os ganhos da intervenção, em proporçóes diferentes frente às variáveis investigadas, são explicados, também, pelo nível de funcionamento de cada participante evidenciado por intermédio do instrumento CARS.

Os resultados deste estudo sugerem a efetividade da IMP no processo de escolarização de alunos com autismo, inseridos nos anos iniciais do Ensino Fundamental, tanto relacionada ao engajamento na tarefa quanto à aprendizagem de habilidades pré-acadêmicas e formais. Se, por um lado, os resultados obtidos variaram de acordo com as especificidades de cada participante; por outro, a intervenção mostrou-se potencialmente significativa no que tange ao ambiente e à dinâmica escolar, apesar das vicissitudes deste espaço.

Cabe ressaltar a influência de variáveis contextuais em que a intervenção se insere, como a natureza das atividades propostas, a organização do ambiente e a característica dos pares, as quais, como os dados desta pesquisa sugerem, podem potencializar ou não os resultados obtidos. Nesta intervenção não só o comportamento mediador dos pares pode auxiliar no engajamento da tarefa, mas também a qualidade da tarefa realizada pelo aluno e da proposta pela professora. Se a atividade não condisser com seu nível de desenvolvimento acadêmico, dificilmente alguma mediação poderá ser eficaz ao ponto de engajar a criança com autismo na tarefa. Nesse sentido, a organização e estruturação do ambiente cumprem, juntamente ao perfil mais interativo dos pares, um papel facilitador das mediaçóes e, consequentemente, dos efeitos sobre as habilidades pretendidas. Destaca-se a importância do professor de sala de aula 
comum no sucesso da intervenção, bem como a necessidade de conhecer as potencialidades, as necessidades e as limitaçóes de seu aluno.

Ao considerar esses aspectos, a IMP tem potencial de contribuição para o desenvolvimento da aprendizagem tanto pré-acadêmica, a qual incide sobre problemas de comportamento aumentando o tempo de engajamento na tarefa, quanto formal, ampliando e auxiliando os alunos na construção de conhecimentos acadêmicos.

Apesar de as contribuições da intervenção terem sido evidenciadas em proporçóes diferentes entre os participantes, a IMP mostrou-se importante para lidar com problemas comportamentais da aluna Maria relacionados à aprendizagem pré-acadêmica, especialmente à agitação apresentada por ela, o que preconiza condiçóes necessárias para o avanço em habilidades acadêmicas formais. Percebe-se que, mesmo com pouca diferença na mediação dos pares, houve diferença estatisticamente significativa no engajamento de Maria. $\mathrm{O}$ resultado pouco incipiente, relacionado a essa variável, tem ligação, entre outros fatores, com o curto tempo de duração da intervenção.

A IMP trouxe resultados para além dos objetivos propostos inicialmente neste estudo. A eficácia da intervenção não se limitou à sala de aula e às atividades de classe, visto que ela pode ser externalizada por meio da presença e interação mais efetiva dos pares em todos os espaços da escola, incluindo recreio, chegada e aulas de educação física, por exemplo. Nesses momentos, pôde-se observar proximidade e mediação maior e mais efetiva dos colegas, tecendo resultados positivos nas atividades por ora desenvolvidas nestes espaços.

Tendo como referência o desempenho dos alunos João e Maria, em todos os protocolos e em todas as testagens aplicadas neste estudo, foi possível verificar que a metodologia quanti-qualitativa foi essencial para aprofundar o estudo das variáveis positivas e negativas que influenciam na mediação e, consequentemente, na aprendizagem desses alunos.

A fidelidade da intervenção realizada nesta pesquisa trouxe diretrizes que podem contribuir para a sua implementação efetiva em escolas brasileiras, cuja validade social indica que a dinâmica e a prática se assemelham ao trabalho realizado nas escolas, não necessitando de mais investimentos para sua implementação.

No que se refere às limitações do estudo, assume-se, ao final desta pesquisa, as restriçôes vivenciadas e percebidas ao longo do seu desenvolvimento. Destaca-se que essas limitaçóes poderiam ter dado novos desdobramentos aos resultados encontrados. Em primeiro lugar, aponta-se a utilização de um delineamento de ensino $\mathrm{AxB}$ com apenas dois participantes. Esse tipo de delineamento e o pequeno número de participantes não viabilizam o controle experimental e não permitem estabelecer relações funcionais de causa e efeito entre as variáveis estudadas, a partir de recomendaçóes mínimas de qualidade metodológica apontadas por Horner et al. (2005). Em segundo lugar, este estudo não conseguiu incorporar as professoras de sala de aula regular no trabalho desenvolvido, ocasionando resultados incipientes relacionados à avaliação do protocolo que exigia continuidade do que estava sendo trabalhado na pré e pós-intervenção. Salienta-se que a presença e a participação efetiva desse profissional nas atividades que envolvem a implementação da intervenção e, posteriormente, a continuidade dos trabalhos desenvolvidos em sala de aula, é essencial para que os resultados sejam satisfatórios. Além disso, 
a presença e a aprovação desse profissional em momentos da mediação, mesmo que por gestos, podem deixar os pares mais seguros, contribuindo no trabalho realizado.

Constatou-se, ainda, na análise dos resultados, a necessidade da construção e do uso de um instrumento específico que pudesse quantificar os dados relacionados à orientação dada aos pares. Assim, seria possível analisar a qualidade da orientação e sua relação com os resultados obtidos, bem como permitiria que o leitor pudesse saber como foi realizada a orientaçáo a partir da análise dos vídeos.

Por último, é necessário levar em conta que o período da realização da coleta de dados deste estudo coincidiu com o término do ano letivo escolar, sendo iniciado em agosto e finalizado em dezembro. Percebeu-se que, por ser final de ano, período de maior fadiga escolar em função da grande demanda nessa época, como elaboração de pareceres, fechamento de cadernos de chamada, atividades extraclasse (como apresentaçóes artísticas relacionadas ao final do ano), a manutenção das filmagens tornou-se desgastante tanto para os professores quanto para a direção da escola. Esse aspecto influenciou a intensidade da intervenção pelo pouco tempo de coleta de dados com os participantes. Controlar e investigar fatores relativos à intensidade da intervenção têm sido apontados como essenciais na literatura (O’Donoghue et al., 2021) para dar suporte às melhores práticas e recomendações de implementação da IMP.

Para os próximos estudos que envolvam a intervenção, além de considerar os aspectos anteriormente mencionados, sugere-se a pontuação separada dos pares que atuam na intervenção. Nos protocolos analisados, ficou-se com a impressão de que talvez um par tenha se engajado muito mais que o outro, refletindo, assim, nos resultados do estudo.

Embora se saiba teoricamente que o efeito da IMP por aproximação seja menor em função de não se ter uma orientação específica sobre a habilidade a ser desenvolvida na criança com autismo, este estudo não contemplou uma linha de base sem a presença física de um par anterior à linha de base com aproximação. Assim, estudos futuros poderão incluir essa variável nas análises.

Somado a isso, cabe destacar que delineamentos de single case research (SCR) não permitem generalizaçóes para uma população de alunos com autismo, o que é possível a partir da replicação de estudos individuais com diferentes participantes e contextos para confirmação, ou não, de que a IMP demonstrou efeito. Nessa perspectiva, este estudo soma-se à vasta literatura internacional, indicando a eficácia da IMP para diversas habilidades sociais, e à escassa literatura quanto às contribuiçóes dessa intervenção para habilidades acadêmicas no contexto inclusivo, sobretudo no cenário educacional brasileiro. No entanto, devido à realidade das escolas brasileiras, no que diz respeito à quantidade de alunos, à precariedade financeira para materiais pedagógicos etc., sugere-se que esta pesquisa seja replicada com um maior número de participantes em diferentes níveis do espectro e de diferentes escolas, com delineamentos experimentais mais robustos, podendo, assim, confirmar os resultados e as conclusóes deste estudo e auxiliar no desenvolvimento tanto de práticas pedagógicas inclusivas como na efetivação da escolarização de alunos com autismo de forma mais coerente com suas especificidades, possibilitando maior oportunidade de sucesso a esses educandos. 


\section{REFERÊNCIAS}

Associação Americana de Psicologia. (2013). DSM-5: Manual Diagnóstico e Estatístico de Transtornos Mentais. Artmed.

Banda, D. R., Hart, S. L., \& Liu-Gitz, L. (2010). Impact of training peers and children with autism on social skills during center time activities in inclusive classrooms. Research in Autism Spectrum Disorders, 4(4), 619-625. https://doi.org/10.1016/j.rasd.2009.12.005

Bastos, J., Esteves, K., Silva, O. A. R., Furlam, N. A. O., Rodrigues C. T., \& Barbosa F. L. A. (2018). Modelação em vídeo e intervenção mediada por pares para promover interações sociais de alunos com Transtorno do Espectro Autista. Acta Comportamentalia, 26(2), 249-266.

Byers, B. J., Reichle, J., \& Symonf, J. (2012). Single-subject experimental design for evidencebased practice. American Journal of Speech Language Pathology, 21(4), 397-414. https://doi. org/10.1044/1058-0360(2012/11-0036)

Camargo, S. P. H., \& Bosa, C. A. (2009). Competência social, inclusão escolar e autismo: revisão crítica da literatura. Psicologia \& Sociedade, 21(1), 65-74. https://dx.doi.org/10.1590/S010271822009000100008

Camargo, S. P. H., Silva, G. L., Crespo, R. A. O., Oliveira, C. R., \& Magalhães, S. L. (2020). Desafios no processo de escolarização de crianças com autismo no contexto inclusivo: diretrizes para formação continuada na perspectiva dos professores. Educação em Revista, 36(e214220), 1-22. https://dx.doi. org/10.1590/0102-4698214220

Carter, E. W., Sisco, L. G., Chung, Y. C., \& Stanton-Chapman, T. L. (2010). Peer interactions of students with intellectual disabilities and/or autism: A map of the intervention literature. Research and Practice for Persons with Severe Disabilities, 35(3-4), 63-79. https://doi.org/10.2511/rpsd.35.34.63

Carvalho. G. B. M. de, Geremia, I., Osowski, V. S., Freitas, L. A. B. de, \& Rocca, J. Z. (2017). Intervenção mediada por pares como estratégia de inclusão de crianças com transtorno de espectro autista. Corixo-Revista de Extensão Universitária, 5, 1-12. https://periodicoscientificos.ufmt.br/ojs/ index.php/corixo/article/view/6473/4218

Chan, J. M., Lang, R., Rispoli, M., O’Reilly, M., Sigafoos, J., \& Cole, H. (2009). Use of peer-mediated interventions in the treatment of autism spectrum disorders: A systematic review. Research in Autism Spectrum Disorders, 3(4), 876-889. https://doi.org/10.1016/j.rasd.2009.04.003

Charlop M. H., Lang R., \& Rispoli, M. (2018). Lights, Camera, Action! Teaching Play and Social Skills to Children with Autism Spectrum Disorder Through Video Modeling. In M. H. Charlop, R. Lang, \& M. Rispoli (Eds.), Play and Social Skills for Children with Autism Spectrum Disorder (pp. 71-94). Evidence-Based Practices in Behavioral Health.

Ferreiro, E., \& Teberosky, A. (1999). Psicogênese da língua escrita. Artmed.

Fialho, J. P. G. (2018). Uso de pistas visuais. In C. P. Duarte, L. C. Silva, \& R. L. Velloso (Eds.), Estratégias da Análise do Comportamento Aplicada para pessoas com Transtorno do Espectro do Autismo (1ª. ed., pp. 195-217). Memnon Ediçôes Científicas.

Ganz, J. B., \& Flores, M. M. (2008). Effects of the use of visual strategies in play groups for children with autism spectrum disorders and their peers. Journal of Autism and Developmental Disorders, 38(5), 926-940. https://doi.org/10.1007/s10803-007-0463-4 
Ganz, J. B., Earles-Vollrath, T. L., Heath, A. K., Parker, R. I., Rispoli, M. J., \& Duran, J. B. (2012). A meta-analysis of single case research studies on aided augmentative and alternative communication systems with individuals with autism spectrum disorders. Journal of Autism and Developmental Disorders, 42(1), 60-74. https://doi.org/10.1007/s10803-011-1212-2

Giaconi, C., \& Rodrigues, M. B. (2014). Organização do espaço e do tempo na inclusão de sujeitos com autismo. Educação \& Realidade, 39(3), 687-705. http://dx.doi.org/10.1590/S217562362014000300004

Gifford-Smith, M. E., \& Brownell, C. A. (2003). Childhood peer relationships: Social acceptance, friendships, and peer networks. Journal of School Psychology, 41(4), 235-284. https://doi. org/10.1016/S0022-4405(03)00048-7

Goldstein, H., Kaczmarek, L., Pennington, R., \& Shafer, K. (1992). Peer-mediated intervention: Attending to, commenting on, and acknowledging the behavior of preschoolers with autism. Journal of Applied Behavior Analysis, 25(2), 289-305. https://doi.org/10.1901/jaba.1992.25-289

Harper, C. B., Symon, J. B., \& Frea, W. D. (2008). Recess is time-in: Using peers to improve social skills of children with autism. Journal of Autism and Developmental Disorders, 38(5), 815-826. https:// doi.org/10.1007/s10803-007-0449-2

Hoff, K. E., \& Robinson, S. L. (2002). Best practices in peer-mediated interventions. In A. Thomas, \& J. Grimes (Eds.), Best Practices in School Psychology IV (v. 2, pp. 1555-1567). APA.

Horner, R. H., Carr, E. G., Halle, J., McGee, G., Odom, S., \& Wolery, M. (2005). The use of singlesubject research to identify evidence-based practice in special education. Exceptional Children, 71(2), 165-179. https://doi.org/10.1177/001440290507100203

Jansen, L. M., Gispen-de Wied, C. C., Van der Gaag, R. J., \& Van Engeland, H. (2003). Differentiation between autism and multiple complex developmental disorder in response to psychosocial stress. Neuropsychopharmacology, 28(3), 582-590. https://doi.org/10.1038/sj.npp.1300046

Kamps, D. M., Leonard, B. R., Vernon, S., Dugan, E. P., Delquadri, J. C., Gershon, B., Wade, L., \& Folk, L. (1992). Teaching social skills to students with autism to increase peer interactions in an integrated first-grade classroom. Journal of Applied Behavior Analysis, 25(2), 281-288. https://doi. org/10.1901/jaba.1992.25-281

Kamps, D., Royer, J., Dugan, E., Kravits, T., Gonzalez-Lopez, A., Garcia, J., Carnazzo, K., Morrison, L., \& Kane, L. G. (2002). Peer training to facilitate social interaction for elementary students with autism and their peers. Exceptional Children, 68(2), 173-187. https://doi. org/10.1177/001440290206800202

Keen, D., Sigafoos, J., \& Woodyatt, G. (2011). Replacing prelinguistic behaviors with functional communication. Journal of Autism and Developmental Disorders, 31, 385-98. https://doi. org/10.1023/A:1010612618969

Lei no 13.146, de 6 de julho de 2015. Institui a Lei Brasileira de Inclusão da Pessoa com Deficiência (Estatuto da Pessoa com Deficiência). http://www.planalto.gov.br/ccivil_03/_Ato2015-2018/2015/ Lei/L13146.htm

Lemos, E. L. D. M. D., Salomão, N. M. R., \& Agripino-Ramos, C. S. (2014). Inclusão de crianças autistas: um estudo sobre interaçóes sociais no contexto escolar. Revista Brasileira de Educação Especial, 20(1), 117-130. https://doi.org/10.1590/S1413-65382014000100009 
Lux, A. L., \& Osborne, J. P. (2004). A proposal for case definitions and outcome measures in studies of infantile spasms and West syndrome: consensus statement of the West Delphi group. Epilepsia, 45(11), 1416-1428. https://doi.org/10.1111/j.0013-9580.2004.02404.x

Martins, M. (2007). Inclusão de alunos autistas no ensino regular: concepçóes e práticas pedagógicas de professores regente [Dissertação de Mestrado, Universidade Católica de Brasília, Faculdade de Psicologia]. Repositório da Universidade Católica de Brasília. https://bdtd.ucb.br:8443/jspui/ handle/123456789/1887

McConnell, S. R. (2002). Interventions to facilitate social interaction for young children with autism: Review of available research and recommendations for educational intervention and future research. Journal of Autism and Developmental Disorders, 32(5), 351-372. https://doi. org/10.1023/A:1020537805154

McCurdy, E. E., \& Cole, C. L. (2014). Use of a peer support intervention for promoting academic engagement of students with autism in general education settings. Journal of Autism and Developmental Disorders, 44(4), 883-893. https://doi.org/10.1007/s10803-013-1941-5

Meadan, H., Ostrosky, M. M., Triplett, B., Michna, A., \& Fettig, A. (2011). Using visual supports with young children with autism spectrum disorder. Teaching Exceptional Children, 43(6), 28-35. https://doi.org/10.1177/004005991104300603

Nunes, D. R., Azevedo, M., \& Schmidt, C. (2013). Inclusão educacional de pessoas com Autismo no Brasil: uma revisão da literatura. Revista Educação Especial, 26(47), 557-572. http://dx.doi. org/10.5902/1984686X10178

Nunes, L., \& Walter, C. (2014). Pesquisa Experimental em educação especial. In L. Nunes (Org.), Novas trilhas no modo de fazer pesquisa em Educação Especial (pp. 27-51). ABPEE/Marquezine Manzini.

O’Donoghue, M., O’Dea, A., O’Leary, N., Kennedy, N., Forbes, J., \& Murphy, C. A. (2021). Systematic Review of Peer-Mediated Intervention for Children with Autism Who Are Minimally Verbal. Review Journal of Autism and Developmental Disorders, 8, 51-66. https://doi.org/10.1007/ s40489-020-00201-2

Odom, S. L., McConnell, S. R., \& Chandler, L. K. (1993). Acceptability and feasibility of classroombased social interaction interventions for young children with disabilities. Exceptional Children, 60(3), 226-236. https://doi.org/10.1177/001440299406000304

Ostrosky, M. M., \& Kaiser, A. P. (1995). The effects of a peer-mediated intervention on the social communicative interactions between children with and without special needs. Journal of Behavioral Education, 5(2), 151-171. https://doi.org/10.1007/BF02110203

Parker, R. I., \& Vannest, K. J. (2012). Bottom-up analysis of single-case research designs. Journal of Behavioral Education, 21(3), 254-265. https://doi.org/10.1007/s10864-012-9153-1

Parker, R. I., Vannest, K. J., Davis, J. L., \& Sauber, S. B. (2011). Combining nonoverlap and trend for single-case research: Tau-U. Behavior Therapy, 42(2), 284-299. https://doi.org/10.1016/j. beth.2010.08.006

Pereira, A. M. (2007). Autismo Infantil: Tradução e validação da CARS (Childhood Autism Rating Scale) para uso no Brasil [Dissertação de Mestrado, Universidade Federal do Rio Grande do Sul]. Repositório LUME. https://www.lume.ufrgs.br/bitstream/handle/10183/12936/000634977.pdf 
Pereira, A. W., Riesgo, M., \& Wagner, A. M. (2008). Autismo infantil: tradução e validação da Childhood Autism Rating Scale para uso no Brasil. Jornal de Pediatria, 84(6), 487-494. https:// dx.doi.org/10.1590/S0021-75572008000700004

Pierce, K., \& Schreibman, L. (1997). Multiple peer use of pivotal response training to increase social behaviors of classmates with autism: Results from trained and untrained peers. Journal of Applied Behavior Analysis, 30(1), 157-160. https://doi.org/10.1901/jaba.1997.30-157

Ramos, F. S., Bittencourt, D. D., Camargo, S. P. H., \& Schmidt, C. (2018). Intervenção mediada por pares: Conceito e implicações para a pesquisa e para as práticas pedagógicas de professores de alunos com autismo. Education Policy Analysis Archives, 26(1), 95-105. https://doi.org/10.14507/ epaa.26.3367

Ramos, F. (2019). Transtorno do Espectro Autista e intervenção instrucional mediada por pares: aprendizagem no contexto de inclusão [Doutorado em Educação, Universidade Federal de Santa Maria]. Repositório da Universidade Federal de Santa Maria. https://repositorio.ufsm.br/handle/1/20485

Rogers, S. J. (2000). Interventions that facilitate socialization in children with autism. Journal of Autism and Developmental Disorders, 30(5), 399-409. https://doi.org/10.1023/A:1005543321840

Rubin, K. H., Bukowski, W. M., \& Laursen, B. (2009). Handbook of peer interactions, relationships, and groups. Guilford Press.

Sam, A., \& Afirm Team. (2015). Visual supports. Chapel Hill, NC: National Professional Development Center on Autism Spectrum Disorder, FPG Child Development Center, University of North Carolina. http://afirm.fpg.unc.edu/visual-supports

Sampaio, A. A. S., Azevedo, F. H. B., Cardoso, L. R. D., Lima, C., Pereira, M. B. R., \& Andery, M. A. P. A. (2008). Uma introdução aos delineamentos experimentais de sujeito único. Interação em Psicologia, 12(1), 151-164. http://dx.doi.org/10.5380/psi.v12i1.9537

Schmidt, C., Ramos, F. S., \& Bittencourt, D. D. (2019). Intervenção mediada por pares como prática pedagógica para alunos com autismo. In A. C. O. Pavão, \& S. M. O. Pavão (Orgs.), Práticas educacionais inclusivas na educação básica (pp. 87-104). FACOS-UFSM.

Strain, P. S., Danko, C. D., \& Kohler, F. (1995). Activity engagement and social interaction development in young children with autism: An examination of "free" intervention effects. Journal of Emotional and Behavioral Disorders, 3(2), 108-123. https://doi.org/10.1023/A:1020537805154

Watkins, L., O’Reilly, M., Kuhn, M., Gevarter, C., Lancioni, G. E., Sigafoos, J., \& Lang, R. (2015). A review of peer-mediated social interaction interventions for students with autism in inclusive settings. Journal of Autism and Developmental Disorders, 45(4), 1070-1083. https://doi.org/10.1007/ s10803-014-2264-x

Recebido em: 30/12/2020

Reformulado em: 27/02/2021

Aprovado em: 16/03/2021 